\title{
CLOSED-FORM GREEN'S FUNCTIONS OF HED, HMD, VED, AND VMD FOR MULTILAYER MEDIA
}

\author{
M. İrşadi Aksun ${ }^{\dagger}$ \\ Bilkent University \\ Dept. of Elect. and Electronics Eng. \\ 06533 Ankara-Turkey
}

\author{
Güllin Dural* \\ Middle East Technical University \\ Dept. of Elect and Electronics Eng. \\ 06531 Ankara-Turkey
}

Abstract

The closed-form Green's functions of the vector and scalar potentials in the spatial domain are presented for the sources of horizontal electric, magnetic, and vertical electric, magnetic dipoles embedded in a general, multilayer, planar medium. The spectral domain Green's functions in an arbitrary layer are obtained through the Green's function of the source layer by using a recursive algorithm. Then, the spatial domain closed-form Green's functions are obtained by adding the contributions of the direct terms, asympthotic components and the complex images approximated by the Generalized Pencil of Function method.

\section{Introduction}

Various studies have been made with layered microstrip st ructures [1-3] due to the increased popularity of the use of multilayer transmission lines such as striplines, covered microstrip lines and suspended substrate lines.

The rigorous analysis of layered structures requires the conputation of the Green's functions for multilayer media, which are usually represented by the Sommerfeld integrals and the closed-form expressions in the spatial and spectral domains, respectively. The numerical evaluation of the matrix elements of the method of noments (MoM) is very time consuming because of the numerical integration of the Sommerfeld integrals in the spatial domain, and slow convergent, highly oscillatory double integrals in the spectral domain. To circumvent this problem, closed-form Green's functions in the spatial domain were formulated for a thick subtrate by using the Sommerfeld identity and the original Prony's method [4]. This technique has further been extended to microstrip geometries with both substrate and superstrate whose thickness can be arbitrary [3].

In this paper, the closed-form Green's functions for the vector and the scalar potentials of a Horizontal Electric Dipole (HED), Horizontal Magnetic Dipole (HMD), Vertical Electric Dipole (VED), and Vertical Magnetic Dipole (VMD) located in an arbitrary layer of a multilayered medium are presented. The spectral domain Green's functions are obtained from the Green's functions of the source layer with the use of an iterative procedure [5]. Then, the contributions of the direct terms and asymthotic terms are added analytically to the terms which are obtained from the approximation of the remaining integrand with the use of the Generalized Pencil of Function (GPOF) method [6].

'supported in part by the NATO SES grant TU-MIMIC.

$0.7803-1246-5 / 93 / \$ 3.00 \bigcirc 1993$ IEEE. 


\section{Theory}

A general multilayer geometry is shown in Fig. 1, where the source (HED, HMD, VED or VMD) is embedded in region $i$. The $z$-dependence of the fields in the source region is written as the sum of the direct terms and up-and down-going waves due to the reflections from the boundarjes at $z=-h$ and $z=d_{i}-h$, respectively. The coefficients of up- and down-going waves can be obtained in terms of the generalized reflection coefficients by applying the appropriate boundary conditions. The spectral domain Green's functions in the source layer (region i) are obtained for a horizontal electric dipole, $\vec{J}^{e}$, and a horizontal magnetic dipole, $\vec{J}^{m}$, as

$$
\begin{aligned}
& \tilde{G}_{x x}^{A}=\frac{\mu_{i}}{2 j k_{z_{1}}}\left[e^{-j k_{z_{i}}|z|}+A_{i}^{e} e^{j k_{z_{1}} z}+C_{i}^{e} e^{-j k_{i} z}\right] \\
& \check{G}_{z x}^{A}=\frac{-\mu_{i}}{2 j k_{z_{i}}}\left[\frac{k_{x} k_{z_{i}}}{k_{\rho^{2}}}\left(A_{i}^{e}+B_{i}^{e}\right) e^{j k_{z_{i}} z}+\frac{k_{x} k_{z_{i}}}{k_{\rho}^{2}}\left(D_{i}^{e}-C_{i}^{e}\right) e^{-j k_{z_{i}} z}\right]
\end{aligned}
$$

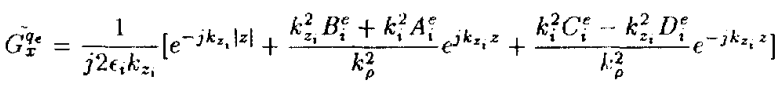

$$
\begin{aligned}
& \tilde{G}_{x x}^{F}=\frac{\epsilon_{i}}{2 j k_{z_{1}}}\left[e^{-j k_{z_{\mathrm{t}}}|z|}+A_{i}^{m} e^{j k_{z_{i} z}}+C_{i}^{m} e^{-j k_{z_{i}} z}\right] \\
& \left.\tilde{G}_{z x}^{F}=\frac{-\epsilon_{i}}{2 j k_{z_{i}}} \frac{k_{x} k_{z_{1}}}{k_{\rho^{2}}}\left(A_{i}^{m}+B_{i}^{m}\right) e^{j k_{z_{i}} z}+\frac{k_{x} k_{z_{i}}}{k_{p}^{2}}\left(D_{i}^{m}-C_{i}^{m}\right) \epsilon^{-j k_{z_{i}} z}\right] \\
& \tilde{G}_{x}^{q_{m}}=\frac{1}{j 2 \mu_{i} k_{z_{i}}}\left[e^{-j k_{z_{i}}|z|}+\frac{k_{z_{i}}^{2} B_{i}^{m}+k_{i}^{2} A_{i}^{m}}{k_{\rho}^{2}} e^{j k_{z_{i}} z}+\frac{k_{i}^{2} C_{i}^{m}-k_{z_{i}}^{2} D_{i}^{m}}{k_{p}^{2}} e^{-j k_{z_{i}} z}\right]
\end{aligned}
$$

where the factors $A_{i}^{e}, B_{i}^{e}, C_{i}^{e}, D_{i}^{e}$ and $A_{i}^{m}, B_{i}^{m}, C_{i}^{m}, D_{i}^{m}$ are functions of the generalized reflection coefficients and the geometry and $k_{i}^{2}=k_{\rho}^{2}+k_{z_{1}}^{2}$. The superscripts $A$ and $F$ represent the magnetic and electric vector potentials, respectively, $q$ represents the scalar potentials, and $e$ and $m$ are used for the electric and magnetic sources, respectively.

For the layers different from the source layer, the amplitudes of the up- and downgoing waves are related to those in the adjacent layers by utilizing an iterative algorithm as

$$
A_{i-m}^{-}=A_{i-m+1}^{-} \frac{T_{i-m+1, i-m} e^{j\left(k_{z_{i}-m+1}-k_{z_{i-m}}\right)\left(-h-z_{-m+1}\right)}}{1-R_{i-m, i-m+1} R_{i-m, i-m-1} e^{-j k_{z_{i-m}}}{ }^{2\left(z_{-m}-z_{-m+1}\right)}}
$$

where $R_{j, k}$ and $\bar{R}_{j, k}$ represent the Fresnel reflection coefficients and the generalized reflection coefficients, respectively, and $T_{j, k}$ is the transmission coefficient. Therefore, starting from the source layer, the field expressions in an arbitrary layer can be calculated iteratively. The closed-form expressions of the spatial domain Green's functions are then obtained by adding the contributions of direct terms and asympthotic components by using the Sommerfeld identity and approximating the remaining integrand by the GPOF method which is more efficient than the Prony's method [7] in terms of its noise sensitivity and the requirement for the additional analytical manipulations. While approximating the integrand by the GPOF method, the $\mathrm{z}$ variaton of $\bar{G}_{z x}$ and $\tilde{G}_{\rho}$ are kept in explicit form for the proper use of the Green's functions in MOM applications. 


\section{Applications}

Various layered microstrip geometries have been studied fo demonstrato the validity of the technique. As a typical example, the closed-form Green's functions for the HED and HMD of a covered microstrip line with an air-gap between the two dielectric substrates are given in Figs. 2(a) and 2(b), respectively. The amplitudes and phases of the Green's functions of the vector potentials $\tilde{G}_{x x}^{A}$ and $\tilde{G}_{x x}^{F}$ calculated using both the closed forms (approximate) and the numerical integration (exact) are shown in Figs. 2(a) and 2(b) for $\epsilon_{\tau_{1}}=\epsilon_{r_{3}}=10.2, d_{1}=d_{3}=0.13 \mathrm{~cm}, \epsilon_{\tau_{3}}=1$ and $d_{2}=0.05 \mathrm{~cm}$. In all the cases studied, the approximate results are found to be in excellent agreement with the exact ones for both vertical ind horizontal sources, and for all the components of the Green's functions.

\section{Conclusions}

The closed-form Green's funcitions in the spatial domain are presented for a general planar, multilayer medium for the HED, HMD, VED and VMD. The computational efficiency in the calculation of the spatial domain Green's functions is significantly increased with the use of the GPOF method. A very good agreement is observed between the approximate and exact Green's functions.

\section{References}

[1] T. Itoh,"Spectral domain immitance approach for dispersion characteristics of generalized printed transmission lines", IEEE, Trans. Microwave Theory and Tech., Vol. MTT 28, No. 7, pp. 733-736, July 1980.

[2] N. K. Das and D. M. Pozar, "A spectral-domain Green's function for multilayer dielectric substrates with application to multilayer transmission lines", IEEE, Trans. Microwave Theory and Tech., Vol. MTT 35, No 3,pp. 326-335, March 1987.

[3] M. I. Aksun and R. Mittra, "Derivation of closed-form, Green's functions for a general microstrip geometry", IEEE, Trans. Microwave Theory and Tech., Vol. MTT 40, No. 11, pp. 2055-2062, November 1992.

[4] Y. L. Chow, J. J. Yang, and D. F. Fang and G. E. Howard, "Closed form spatial Green's function for the thick substrate", IEEE, Trans. Microwave Theory and Tech., Vol. MTT 39, No. 3,pp. 588-592, March 1991.

[5] W. C. Chew, Waves and Fields in Inhomogenous Media, Van Nostrad Rein hold, New York 1990.

[6] Y. Hua and T. K. Sarkar, "Generalized pencil-of-function method for extracting poles of an EM system from its transient response", IEEE Trans. Antennas and Prop., Vol. Ap-37, No.2, pp.229-234, February 1989.

[7] S. L. Marple, Digital Spectral Analysis with Applications, Englewood Cliffs, New Jersey, Prentice Hall, 1987. 

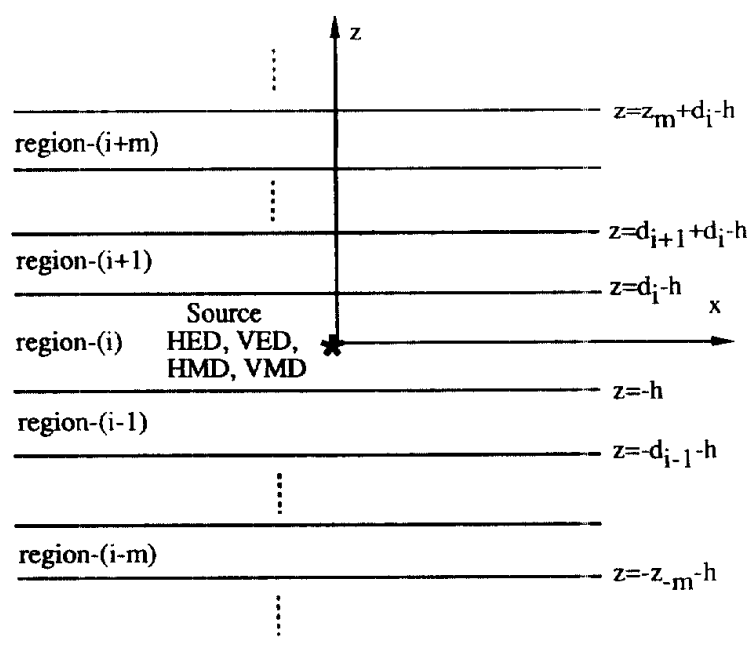

Fig. 1. A source embedded in a multilayered medium

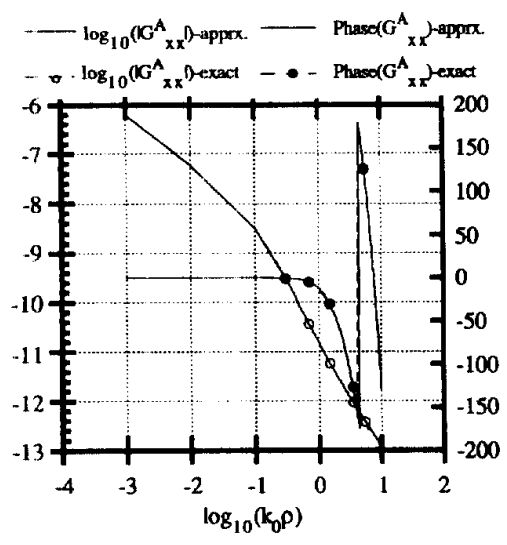

(a)

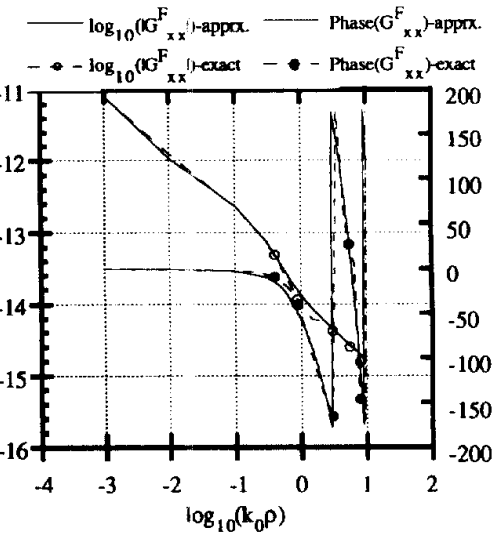

(b)

Fig. 2. The Green's functions for the horizontal (a) electric and (b) magnetic potentials in the source layer $(i=2)$; magnitude and phase. $f=3 \mathrm{GHz}$, $\varepsilon_{\mathrm{r} 1}=\varepsilon_{\mathrm{r}}=10.2, \varepsilon_{\mathrm{r} 2}=\varepsilon_{\mathrm{r} 4}=1.0, \mathrm{~d}_{1}=\mathrm{d}_{3}=0.13 \mathrm{~cm}, \mathrm{~d}_{2}=0.05 \mathrm{~cm}$, layer -0 is $P E C$ 